\title{
Retrato da atenção básica no Brasil: gastos e infraestrutura em municípios brasileiros
}

\author{
A portrait of Brazilian primary care: municipal expenditure \\ and infrastructure in Brazilian municipalities
}

Helder Kiyoshi Kashiwakura (https://orcid.org/0000-0002-8187-6447) ${ }^{1}$ Andréa de Oliveira Gonçalves (https://orcid.org/0000-0001-7514-8139) ${ }^{2}$ Ricardo Rocha de Azevedo (https://orcid.org/0000-0001-6302-0760) ${ }^{3}$ André Nunes (https://orcid.org/0000-0001-9928-6245) ${ }^{2}$

César Augusto Tibúrcio Silva (https://orcid.org/0000-0002-5717-9502) ${ }^{2}$

${ }^{1}$ Departamento de Ciências Contábeis e Atuariais, Universidade de Brasília. Campus Universitário Darcy Ribeiro, Prédio da FACE, Asa Norte. 70910970. Brasília DF Brasil. hkiyoshi@yahoo.com.br ${ }^{2}$ Programa de Pós-

Graduação em Ciências Contábeis, Universidade de Brasília. Brasília DF Brasil.

${ }^{3}$ Faculdade de Ciências Contábeis, Universidade Federal de Uberlândia. Uberlândia MG Brasil.

\begin{abstract}
The paper presents the relationship between the allocation of financial resources and the type of local basic health infrastructure in an initial sample of 5,570 Brazilian municipalities. This is an explanatory research using multiple correspondence analysis and quantile regression between expenditure/control variables and the type of structure of PHC Units. The correspondence between the type and the representative variables of expenditure shows that inferior typologies are related to lower per capita expenditure in these variables, and vice versa. Quantile regression showed a positive relationship with the type of infrastructure in the two cycles evaluated. There is evidence that expenditure variables are positively related to infrastructure, which allows us to understand that allocating more resources leads to better infrastructure. Results point to the need to improve the governance of financial resources for health, as municipalities with lower socioeconomic indicators have an infrastructure in the lower categories. We can conclude that there are multiple actors, and the various criteria for allocating and decentralizing resources bring about difficulties of coordination and integration between the entities, restricting the appropriate prioritization in the distribution of resources.
\end{abstract}

Key words Primary health care, Health expenditures, Health infrastructure, Governance
Resumo $O$ trabalho apresenta a relação entre a alocação de recursos financeiros e a tipologia da infraestrutura básica local de saúde em amostra inicial de 5.570 municípios do Brasil. Trata-se de uma pesquisa explicativa com uso da análise de correspondência múltipla e regressão quantílica entre variáveis de gastos/controle e a tipologia da estrutura das Unidades Básicas de Saúde. As correspondências entre a tipologia e variáveis representativas dos gastos mostram que tipologias inferiores estão relacionadas com menores gastos per capita nessas variáveis, e vice-versa. A regressão quantílica apresentou relação positiva com a tipologia da infraestrutura nos dois ciclos avaliados. Há evidência das variáveis de gasto estarem relacionadas, de maneira positiva, à infraestrutu$r a$, permitindo entender que alocar mais recursos leva a melhor infraestrutura. No entanto, há a necessidade de melhoria na governança dos recursos financeiros da saúde, pois municípios com indicadores socioeconômicos inferiores têm infraestrutura nas categorias inferiores. Conclui-se que há grande multiplicidade de atores e os múltiplos critérios para alocação e descentralização de recursos trazem dificuldades de coordenação e integração entre os entes, restringindo a adequada priorização na alocação dos recursos.

Palavras-chave Atenção primária à saúde, Gastos em saúde, Infraestrutura sanitária, Governança 


\section{Introdução}

A criação do Sistema Único de Saúde (SUS), nos anos 1990, levou a atenção básica (AB) a desempenhar importante papel no sistema universal de saúde no Brasil. Nos últimos anos, mesmo com todo o progresso na saúde, a operação desses serviços ainda não é satisfatória, devido às desigualdades regionais, ao desperdício de recursos na prestação de serviços, à subutilização dos leitos hospitalares e ao gasto pouco eficaz dos recursos públicos ${ }^{1}$. Nesse sentido, a gestão local da saúde desempenha papel central e estratégico na antecipação da resolução de problemas, pois a saúde é também um indutor para o desenvolvimento de uma nação. Além de ser um direito essencial, financiado em grande parte por recursos públicos. Assim, ao pensar a alocação dos recursos, é necessário observar se essa é eficiente, eficaz e efetiva, já que a prestação desse tipo de serviço representa a porta de entrada no SUS, ao enfatizar a oferta de serviços por meio de um modelo orientado pela demanda como um fator de redução de custos e de integralidade das ações de saúde ${ }^{2}$.

No âmbito do sistema de saúde, a $\mathrm{AB}$ é prioridade, já que as Unidades Básicas de Saúde (UBS) podem ser o principal equipamento de saúde em nível local, capaz de resolver até $85 \%$ dos problemas de saúde da comunidade 3 . Assim, uma das formas de se efetuar esse tipo de análise é relacionar a composição dos equipamentos básicos de saúde locais aos recursos alocados.

Devido ao crescimento de gastos com saúde, a alocação de recursos públicos tem sido alvo de preocupação de natureza acadêmica e orçamentária em muitos países, inclusive o Brasil. Segundo a Organização Pan-Americana de Saúde ${ }^{4}$, os gastos com saúde representavam 3\% do PIB mundial em 1948 e passaram para 8,7\% do PIB em $2004^{4,5}$.

Diante desse cenário, é importante saber se os recursos descentralizados refletem a infraestrutura disponibilizada para os usuários. Embora pareça evidente que um maior nível de gasto gere maiores investimentos em infraestrutura, essa relação não é necessariamente clara em países em desenvolvimento ${ }^{6,7}$. Se por um lado há significativa correlação entre o aumento das despesas com a atenção básica e o melhor acesso à saúde ${ }^{6}$, por outro, países que ainda não atingiram a maturidade em termos de governança dos recursos públicos não apresentam forte correlação nesse aspecto, devido à existência de outros fatores que interferem na gestão do sistema de saúde ${ }^{7}$.
Sob esse enfoque, este artigo apresenta resultados de pesquisa nacional realizada entre 2016 e 2019, intitulada Retrato da atenção básica no Brasil, sob a perspectiva da governança orçamentária. Particularmente, analisa-se a relação entre o comportamento da alocação dos recursos financeiros públicos em saúde e a tipologia da infraestrutura básica local de saúde nos municípios brasileiros. A hipótese diretriz formulada sugere que o maior dispêndio de recursos financeiros per capita leva a uma melhor infraestrutura dos equipamentos locais de saúde.

É interessante observar as diferentes interpretações da abrangência e do escopo da atenção básica que levaram à utilização de termos distintos para a forma de organizar os sistemas de serviços de saúde nos diversos países. Optou-se aqui por manter os termos originalmente utilizados em cada trabalho citado. No entanto, nesta pesquisa, o termo atenção básica é empregado por conta dos documentos utilizados pelo Ministério da Saúde no Programa Nacional de Melhoria do Acesso e da Qualidade da Atenção Básica (PMAQ).

Observa-se que a qualidade da governança dos recursos públicos é essencial para o desenvolvimento ${ }^{8}$ de uma nação, sobretudo em países com recursos escassos e resultados pobres de gestão de políticas públicas, como o Brasil, dada a evidência de que as instituições orçamentárias interferem substancialmente em áreas como a AB. Isso explica a maior ou menor efetividade das despesas públicas na provisão de melhores condições sociais.

Em estudo ${ }^{9}$ que estimou o efeito dos gastos no desempenho da $\mathrm{AB}$ e a eficiência econômica, evidenciou-se que nos municípios brasileiros a $\mathrm{AB}$ tem melhor desempenho do que os demais níveis de cuidado (média e alta complexidade), o que leva a concluir que há relação positiva entre gasto e desempenho da $\mathrm{AB}$, mas há grandes disparidades regionais em relação ao gasto e ao desempenho da $\mathrm{AB}$ no Brasil9.

Estudo realizado em $2007^{10}$ apresentou uma análise, a partir de categorias dos municípios paulistas, de gastos em saúde com recursos próprios por habitante, transferências do SUS por habitante, tipologia da estrutura do SUS e índice de esforço da AB. Nesse estudo, observou-se que os municípios com maior índice de esforço em $A B$ eram os que apresentavam maior valor de despesa com recursos próprios por habitante e que possuíam estruturas mais simples de atendimento à saúde, o que indica que investem, predominantemente, nas ações de promoção e prevenção da saúde ${ }^{10}$. 
Quanto à infraestrutura, há pesquisas que estabelecem uma tipologia da infraestrutura de saúde dos municípios brasileiros ${ }^{11}$ e outras que tratam da infraestrutura de saúde ${ }^{12-14}$. Esses trabalhos analisaram a distribuição e a qualidade da infraestrutura básica de saúde disponível em regiões específicas, porém nenhum deles estabeleceu uma relação da tipologia da infraestrutura com os gastos públicos.

Dessa forma, apenas o aumento de gastos públicos não é suficiente para a melhoria dos resultados nos serviços de saúde ${ }^{15}$, pois é difícil encontrar relações consistentes entre aumento nos gastos e resultados que demonstrem a importância de se alocar mais recursos para indivíduos de baixa renda. Assim, a infraestrutura de instalações de saúde é um componente importante de um sistema de saúde e não deve ser subestimado ${ }^{16}$.

Há também um estudo que analisou os gastos em saúde de 2001 a 2006 com relação ao percentual do PIB per capita e evidenciou que o Brasil gasta mais em saúde que outros países vizinhos com maiores níveis de renda ${ }^{17}$. Entretanto, conforme os autores, a análise dos indicadores de saúde da população brasileira em comparação aos outros países, que resultou em indicadores piores ${ }^{17}$, gerou a falsa impressão de que o país estivesse investindo suficientemente em saúde para suas possibilidades econômicas. Tais resultados, observados na Tabela 1, não se alteraram substancialmente de 2014 a 2017. Utilizando-se outro indicador de resultado na saúde (morte entre 15 e 60 anos), verifica-se que o Brasil não difere muito de outros países em termos de gasto total per capita e percentual do PIB. Mesmo que alguns países tenham melhorado no quesito alocação de recursos, o Brasil gasta mais, proporcionalmente ao PIB, do que Uruguai, Chile e Colômbia, por exemplo. No entanto, o índice de mortalidade no Brasil continua bem pior do que nesses países.

O Banco Mundial ${ }^{15}$ já havia apontado na mesma direção: ao comparar gasto e indicadores de saúde, como expectativa de vida, mortalidade infantil e mortalidade materna, o Brasil revela um nível intermediário de desempenho entre os países de renda média e na América Latina. Alguns países (Tabela 1) apresentam desempenho melhor do que outros com níveis semelhantes de gasto e desenvolvimento econômico, o que sugere que fatores adicionais podem influenciar a efetividade do gasto público na saúde - como políticas que o direcionam às necessidades da população mais pobre - e a melhor qualidade do gasto, que poderia gerar melhoria dos resultados na área de saúde. Um aspecto evidenciado é que valores mais altos de gastos em saúde em níveis de alta complexidade podem ter pouco impacto nos indicadores gerais de saúde ${ }^{18}$.

\section{Materiais e métodos}

A respeito da alocação de recursos, fez-se uso de dados de acesso público que tratam de despesas com ações e serviços públicos de saúde (ASPS) gerenciados pelo Ministério da Saúde (MS) por meio do SIOPS/DATASUS para 5.570 municípios, classificados por Execução Financeira por Bloco (exercícios financeiros de 2007 a 2017), Classificação Econômica da Despesa (de 2002 a 2017) e Subfunção da Despesa (de 2002 a 2017), conforme previsto na cartilha do Conselho $\mathrm{Na}$ cional de Secretários de Saúde.

A base de dados sobre infraestrutura compõese de dados sobre as UBS, disponibilizados pelo PMAQ, referentes ao primeiro ciclo de avaliação (2012), no total de 38.812 UBS, em 5.543 municípios $^{11}$, e ao segundo ciclo de avaliação (2014), totalizando 24.997 UBS em 5.072 municípios.

$\mathrm{Na}$ análise da composição dos equipamentos básicos de saúde locais, estabeleceu-se uma classificação para a infraestrutura de saúde disponibilizada, que é a sua tipologia ${ }^{11}$, originada do PMAQ, referentes ao primeiro e segundo ciclos, disponibilizados pelo Departamento da Atenção Básica do Ministério da Saúde. Na definição da tipologia, foram adotadas cinco classificações: (1) reprovada, que representa ausência de estrutura de serviço de saúde. São UBS que não cumprem requisitos mínimos na prestação de atendimento ${ }^{11}$; (2) rudimentar, apresentam importante insuficiência de equipes, do elenco de profissionais, de serviços disponíveis e de equipamentos e insumos. São UBS que sequer realizam atendimento ao grupo materno-infantil e tampouco poderiam ser consideradas prestadoras de uma atenção primária seletiva ${ }^{11}$; (3) restrita, caracterizada pela insuficiência de equipamentos e de equipe de saúde. São UBS que necessitam, principalmente, de investimentos em infraestrutura geral e na prestação de serviços de saúde bucal ${ }^{11}$; (4) regular, UBS com equipes de saúde da família e saúde bucal que, com baixo investimento para a melhoria da infraestrutura de equipamentos e insumos, alcançariam o padrão de referência. Por possuírem dificuldade de acesso à internet, podem requerer investimentos em telecomunicações ${ }^{11}$; (5) padrão de referência, UBS que dispõem de condições elementares para funcionamento e prestação de escopo de ações em $A B$. Funcionam cinco ou mais dias na semana 


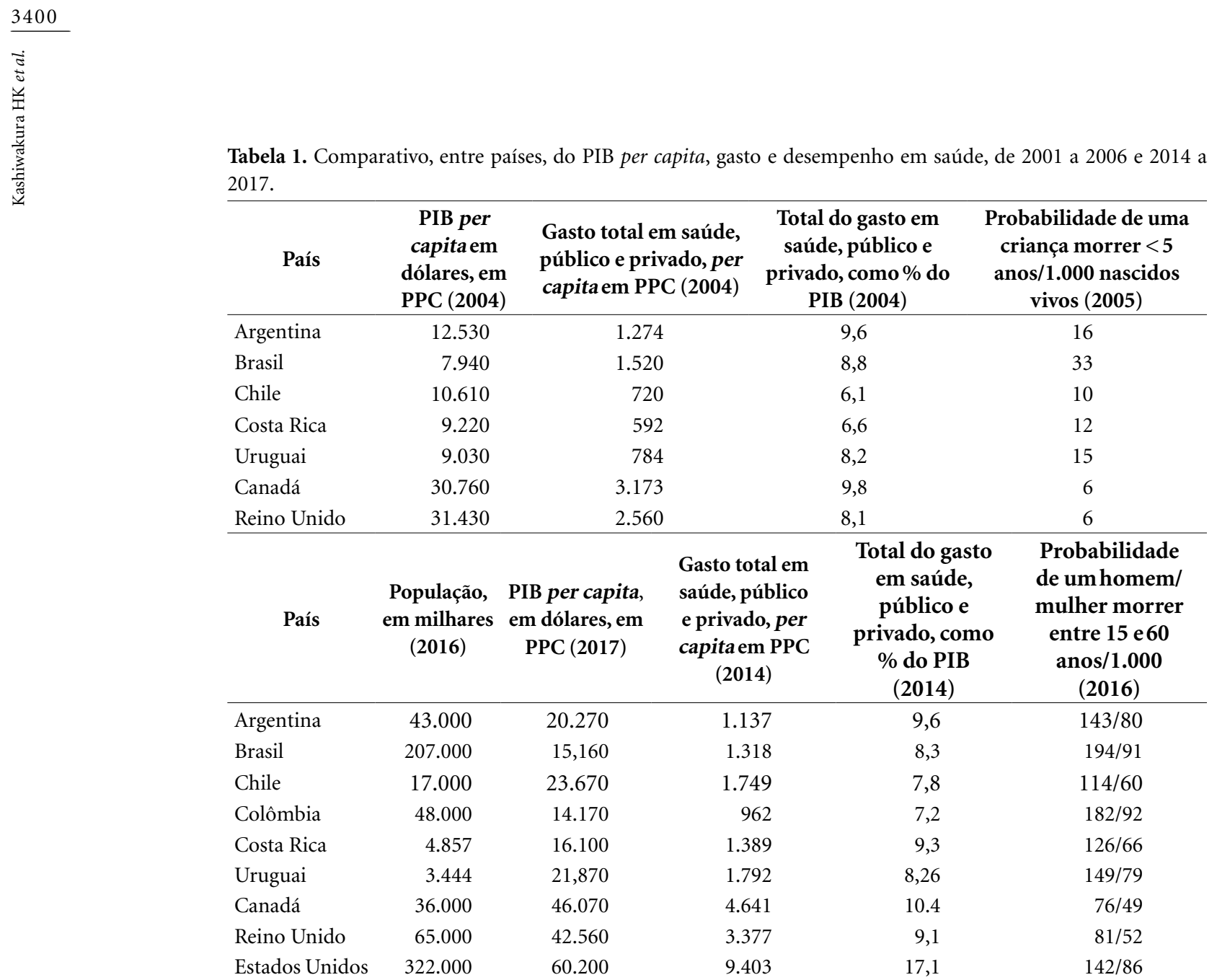

Fonte: Ferrraz e Vieira ${ }^{17}$ (2001 a 2006); elaborado pelos autores, com base em World Bank Atlas (2014 a 2017).

Disponível em: https://openknowledge.worldbank.org/handle/10986/26306; e World Health Organization (WHO). Disponível em: http://www.who.int/countries/en/. Acesso em: 22 dez. 2017.

em dois ou três turnos de atendimento e ofertam consultas médicas, enfermagem e odontologia ${ }^{11}$.

Os dados orçamentários/financeiros referentes à alocação de recursos foram atualizados monetariamente pelo IPCA (Índice de Preços ao Consumidor Amplo, medido pelo IBGE), ano base 2017. Para a análise dos gastos foram calculados valores per capita para cada montante anual nos municípios, segundo dados da população local, disponibilizados pelo IBGE, do período de 2002 a 2017 . O cálculo dos valores per capita (_cpt) foi obtido da divisão do montante alocado anualmente em cada município pela população existente em cada ano (censo ou estimativa do IBGE). As variáveis foram dispostas em três blocos: categoria econômica, subfunção e bloco de gastos (Quadro 1). Em seguida, realizou-se o teste qui-quadrado para esclarecer se a distribuição das variáveis por categoria foi aleatória ou se ocorreu um padrão determinado por interdependência entre as variáveis.

No primeiro momento, realizou-se a análise de correspondência $(\mathrm{AC})^{19-21}$ por pares de vari- áveis, em que foram analisados os gráficos com melhor representatividade, isto é, melhor correspondência entre pares de categorias de variáveis, sendo consolidada em tabelas de correspondência de categorias de variáveis. Essa análise foi utilizada para complementar as evidências não passíveis de ser verificadas na análise de regressão múltipla, pois os pressupostos desse tipo de técnica são mais rigorosos que os daquela.

No segundo momento, utilizou-se a análise de regressão quantílica (RQ) $)^{22-24}$ para identificar relações entre as variáveis independentes (gastos) e a tipologia das UBS (escore das UBS) a partir da definição da tipologia e da composição dos gastos alocados na $\mathrm{AB}$, a fim de verificar como cada variável de gastos influi na tipologia das UBS. Utilizou-se, ainda, outras variáveis de controle, como o índice de desenvolvimento humano municipal (IDHM), o índice de desenvolvimento da saúde (IDS) e o PIB per capita.

Observa-se que a RQ não requer a pressuposição de erros homocedásticos, podendo ser 
Quadro 1. Variáveis sobre gastos.

\begin{tabular}{|l|l|l|}
\hline \multicolumn{1}{|c|}{ Grupos } & \multicolumn{1}{|c|}{ Código da variável } & \multicolumn{1}{c|}{ Conteúdo da variável } \\
\hline \multirow{5}{*}{ Categoria econômica } & desp_pess_enc_cpt & Despesa de pessoal per capita \\
\cline { 2 - 3 } & odc_cpt & Outras despesas correntes per capita \\
\cline { 2 - 3 } & desp_cap_cpt & Despesa de capital per capita \\
\cline { 2 - 3 } & invest_cpt & Investimento per capita \\
\cline { 2 - 3 } Subfunção & desp_geral_cpt & Despesa geral per capita \\
\hline \multirow{5}{*}{ Bloco de gastos } & liq_sub_adm_cpt & Subfunções administrativas per capita \\
\cline { 2 - 3 } & liq_sub_vinc_cpt & Subfunções vinculadas per capita \\
\cline { 2 - 3 } & liq_atbas_cpt & Despesa com atenção básica per capita \\
\cline { 2 - 3 } & liq_desp_tot_cpt & Despesa total per capita \\
\hline & atbas_fed_cpt & Despesa com atenção básica federal per capita \\
\cline { 2 - 3 } & atbas_est_cpt & Despesa com atenção básica estadual per capita \\
\cline { 2 - 3 } & atbas_rprop_cpt & Despesa com atenção básica municipal per capita \\
\cline { 2 - 3 } & atbas_tot_cpt & Despesa total com atenção básica per capita \\
\cline { 2 - 3 } & tot_fed_cpt & Despesa federal total per capita \\
\cline { 2 - 3 } & tot_est_cpt & Despesa estadual total per capita \\
\cline { 2 - 3 } & tot_rprop_cpt & Despesa municipal total per capita \\
\cline { 2 - 3 } & tot_tot_cpt & Despesa total per capita \\
\hline
\end{tabular}

Fonte: Elaborado pelos autores.

utilizada quando a distribuição dos erros é heterocedástica, sendo, ainda, uma técnica robusta com relação à presença de outliers. Possui também uma representação de programação linear, o que facilita a estimação dos parâmetros ${ }^{22-24}$. Além disso, a variável dependente escore_final apresenta distribuição não normal (assimetria negativa), o que faz com que a técnica de regressão quantílica seja a mais adequada quando se trabalha com dados não normais e com presença de heterocedasticidade ${ }^{22-24}$.

Cinco modelos de regressão foram testados, por meio do software Stata 13.0, dividindo-se as variáveis independentes pelo tipo de classificação da despesa (orçamentária e de saúde). O modelo por categoria econômica (RQ1) permite verificar o efeito comparativo de cada tipo de gasto, por exemplo se o gasto com pessoal possui mais influência sobre a tipologia do que as despesas correntes em geral. O modelo por subfunção (RQ2) possibilita observar o efeito de uma subfunção específica, por exemplo se o gasto com $A B$ possui mais influência sobre a tipologia do que o gasto com atenção de média e alta complexidades. $\mathrm{O}$ modelo por blocos de gastos (RQ3, RQ4 e RQ5) permite verificar qual esfera possui gasto com maior efeito sobre a tipologia, por exemplo. Realizaram-se também os testes Jarque-Bera, para a normalidade dos resíduos, o teste VIF (fator de inflação da variância), para a multicolinearidade entre variáveis independentes, e o teste de correlação entre variáveis, para cada modelo.

\section{Resultados e discussão}

Os resultados mostraram que grande parte das UBS - participantes do PMAQ - está classificada nas tipologias 4 (51,2\% no ciclo 1 e $76,8 \%$ no ciclo 2), ou seja, são consideradas regulares, o que significa que essas UBS possuem equipes de saúde da família e saúde bucal que, com melhoria da infraestrutura, alcançariam o padrão de referência $5^{11}$. Isso mostra que mais de $75 \%$ das UBS possuem pequenas deficiências, restando verificar as relações dessa tipologia com os gastos e como se encontra no país.

Ressalte-se, contudo, no Quadro 2, a ocorrência de importante alteração na classificação das UBS: a tipologia 3 (restrita), que apresenta insuficiência de equipamentos e equipes de saúde, teve decréscimo de $26,7 \%$ para $17,1 \%$, enquanto a tipologia 4 (regular), que possui equipes de saúde da família e saúde bucal que, com uma melhora da infraestrutura, alcançariam o padrão de referência, teve acréscimo de 51,79\% para 76,8\%. Essa evolução na classificação da tipologia reflete maior disponibilização de equipamentos e de equipes de saúde, não disponíveis na tipologia 3 (restrita). Importa evidenciar como se dá a distribuição das UBS ti- 
pos 1 (reprovada) e 2 (rudimentar), nos ciclos 1 e 2 , tomando-se por base os estados da federação e levando-se em conta as regiões geográficas do país.

Os resultados corroboram outros estudos, mesmo com metodologias diferentes, no sentido de que a alocação de mais recursos por si só não é suficiente ${ }^{25,26}$. A análise dos gastos anuais per capita por municípios evidenciou municípios que alocam valores inferiores a $\mathrm{R} \$ 50,00$ e outros que alocam valores superiores a $R \$$ 3.500,00. A análise da média de gastos por estado e da média dos escores demonstrou que nem sempre o estado que apresenta a maior média de gastos na $\mathrm{AB}$ tem a maior média de escore final, a exemplo de Pará, Pernambuco, Paraná e São Paulo, o que pode ser visto no Quadro 2, que expõe a comparação da média de gastos alocados na $\mathrm{AB}$ por município e o escore final, para o ciclo 2. A média dos escores do Pará se aproxima de São Paulo, enquanto a média de Pernambuco é ainda maior, embora o montante alocado por aqueles estados não chegue a 1/3 do valor médio alocado nos municípios de São Paulo, evidenciando que há aspectos relacionados à governança que precisam ser considerados.

$\mathrm{Na}$ infraestrutura da $\mathrm{AB}$ e na distribuição de recursos não são cumpridos os mandamentos definidos pela Constituição de 1988 para a perfeita coordenação e integração dos sistemas de saúde, como preconiza a governança orçamentária ${ }^{27-29}$. Observou-se que a $\mathrm{AB}$ não facilita o acesso uni- versal ao diversificado escopo de serviços, de forma coordenada e contínua, mediante a aplicação eficiente de recursos e a prestação de cuidados de alta qualidade, que contribuem para a equidade em saúde ${ }^{30}$, pois uma $\mathrm{AB}$ equipada com UBS dos tipos 1 e 2 não está habilitada a coordenar os serviços de saúde da região e a resolver os problemas da população adscrita.

Observa-se que a Figura 1 evidencia a tipologia da infraestrutura por meio da distribuição da média dos escores dos municípios por estado, indicando que a região Norte, de maior área geográfica e população dispersa espacialmente, apresenta os piores índices socioeconômicos, com a pior média de escore por estado (inferior a 0,85), enquanto as regiões Centro-Oeste e Sul apresentam as melhores médias por estado (superiores a 0,85$)$.

A partir dos resultados acerca da infraestrutura, caracteriza-se a relação entre a estrutura de gastos (variáveis de despesas) e a tipologia da infraestrutura básica local de saúde (UBS) por meio da análise de correspondência $(\mathrm{AC})$ entre as variáveis e da análise de regressão quantílica (RQ).

A análise das evidências com a variável população revelou comportamento homogêneo dos dados observados tanto no ciclo 1 (2012) como no ciclo 2 (2014), permitindo-se afirmar que: municípios com menor porte populacional gastam mais recursos per capita com a $\mathrm{AB}$, têm maiores gastos per capita federais na $\mathrm{AB}$ e maio-

Quadro 2. Evolução da tipologia Unidade Básica de Saúde, gastos na atenção básica e tipologia - Brasil.

\begin{tabular}{|c|c|c|c|c|c|}
\hline \multicolumn{6}{|c|}{ Evolução da tipologia Unidade Básica de Saúde } \\
\hline \multirow{2}{*}{ Tipologia } & \multirow{2}{*}{ Escore } & \multicolumn{2}{|l|}{ Ciclo 1} & \multicolumn{2}{|c|}{ Ciclo 2} \\
\hline & & Número de UBS & $\%$ & Número de UBS & $\%$ \\
\hline 1 - Reprovado & \multirow{5}{*}{$\begin{array}{l}<0,250 \\
\geq 0,250 \text { e }<0,5 \\
\geq 0,5 \text { e }<0,75 \\
\geq 0,75 \text { e }<1,0 \\
=1,0\end{array}$} & 1.690 & 4,3 & 445 & 1,8 \\
\hline 2 - Rudimentar & & 3.639 & 9,3 & 138 & 0,6 \\
\hline 3 - Restrita & & 10.364 & 26,7 & 4.200 & 17,1 \\
\hline 4 - Regular & & 20.102 & 51,7 & 18.816 & 76,8 \\
\hline 5 - Padrão de referênia & & 3.017 & 7,7 & 900 & 3,7 \\
\hline \multicolumn{2}{|c|}{ Total } & 38.812 & 100 & 24.499 & 100,0 \\
\hline \multicolumn{6}{|c|}{ Escore final de gastos na atenção básica e tipologia } \\
\hline \multicolumn{2}{|l|}{ Estado } & \multicolumn{2}{|c|}{$\begin{array}{c}\text { Gastos na } \mathrm{AB} \\
\text { (média per capita anual, em } \mathrm{R} \$ \text { ) }\end{array}$} & \multicolumn{2}{|l|}{$\begin{array}{l}\text { Escore final } \\
\text { (média) }\end{array}$} \\
\hline \multicolumn{2}{|l|}{ Pará } & \multicolumn{2}{|l|}{207} & \multicolumn{2}{|l|}{0,83} \\
\hline \multicolumn{2}{|l|}{ Roraima } & \multicolumn{2}{|l|}{219} & \multicolumn{2}{|l|}{0,66} \\
\hline \multicolumn{2}{|l|}{ Pernambuco } & \multicolumn{2}{|l|}{233} & \multicolumn{2}{|l|}{0,88} \\
\hline \multicolumn{2}{|l|}{ Paraná } & \multicolumn{2}{|l|}{571} & \multicolumn{2}{|l|}{0,85} \\
\hline \multicolumn{2}{|l|}{ São Paulo } & \multicolumn{2}{|l|}{722} & \multicolumn{2}{|l|}{0,84} \\
\hline
\end{tabular}




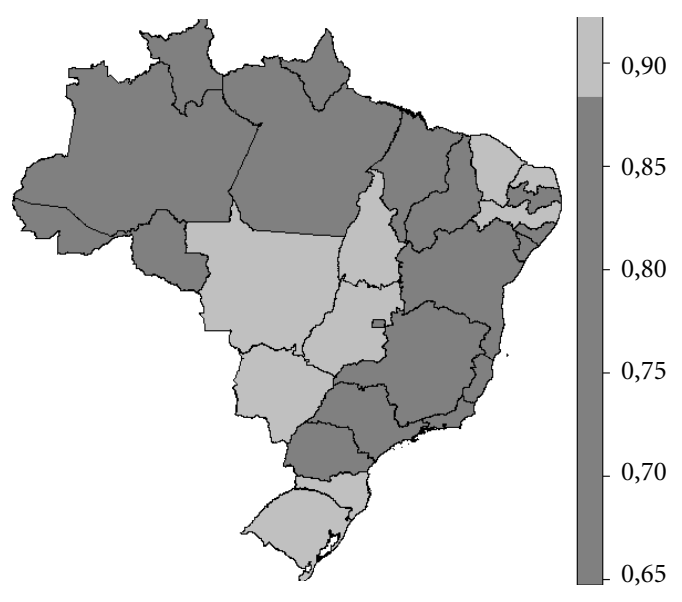

Figura 1. Distribuição da tipologia por média de escore das UBS por estado (ciclo 2).

Legenda: A escala de cores à direita representa a média dos escores das UBS dos municípios por estado.

Fonte: Elaborado pelos autores, 2019.

res gastos per capita com recursos próprios dos municípios na AB. No entanto, possuem menores gastos federais totais per capita. Já nas variáveis de controle há evidências de menores índices de PIB, IDHM, índice de Gini de renda e mortes evitáveis de 5 a 74 anos. No entanto, no ciclo 1 (2012) não foi comprovada relação entre tipologia e população do município.

Relacionado aos resultados com a variável tipologia, a análise das evidências revelou comportamento homogêneo, porém em menor proporção de variáveis observadas, ao se comparar o ciclo 1 (2012) com o ciclo 2 (2014), a saber: os municípios com tipologias inferiores possuem menores gastos federais per capita na $\mathrm{AB}$, menores gastos totais per capita com saúde e menores gastos federais totais per capita com saúde.

No entanto, as seguintes relações só se verificaram no ciclo 1: menores gastos per capita com recursos próprios na saúde e menores gastos per capita com pessoal e encargos. Quanto às variáveis de controle, possuem índices menores de PIB e IDHM no ciclo 1.

Ao se discutir os resultados apresentados por meio da análise de correspondência, verifica-se a convergência para a hipótese diretriz, de que o maior dispêndio de recursos financeiros per capita leva a uma melhor infraestrutura dos equi- pamentos locais de saúde. A convergência dos resultados com a hipótese é corroborada pelas correspondências entre a tipologia e as variáveis representativas dos gastos em saúde realizados nos municípios (gastos federais na $\mathrm{AB}$, gastos federais totais em saúde e gastos totais em saúde), que evidenciam que as tipologias reprovada (1) e rudimentar (2) estão relacionadas com menores gastos per capita.

Acerca do comportamento dos gastos em relação ao porte populacional, os gastos totais federais per capita em saúde são menores em municípios de menor porte, o que se explica pela predominância da $\mathrm{AB}$ nesses municípios. Quanto à tipologia, municípios com tipologias reprovada (1) e rudimentar (2) possuem menores gastos federais na $\mathrm{AB}$, aspecto que merece atenção do governo federal, responsável pelas ações estratégicas, principalmente quando se observa a necessidade de combate às desigualdades regionais e à pobreza, como define a Constituição Federal.

Os resultados evidenciados pela variável gastos totais municipais (recursos próprios) são coerentes com os apresentados em estudo anterior ${ }^{10}$, pois os municípios com maior índice de esforço em $\mathrm{AB}$ apresentaram maior valor de despesa com recursos próprios por habitante e correspondiam àqueles com estruturas mais simples de atendimento à saúde, o que indica investimento predominante em ações de promoção e prevenção da saúde. De certa forma, corroboram com a literatura ao defender que os serviços públicos alcancem os mais pobres. A questão é: como os governos podem garantir a distribuição equitativa dos benefícios dos serviços públicos a quem deles realmente necessita? Assim, dois fatores desempenham papel importante: (1) quando o investimento é colocado em serviços básicos, como cuidados básicos de saúde e educação primária, o número de pobres beneficiados aumenta consideravelmente; e (2) quando o investimento é usado para fornecer níveis mais altos de serviço, como atenção à saúde de alta complexidade, o número de pobres que se beneficia cai. A distribuição de gastos no nível primário é muito menos desigual ${ }^{31}$.

Ao observar a alocação de recursos e a infraestrutura, a situação é diferente, pois as regiões com condições socioeconômicas inferiores têm infraestrutura também inferior. $\mathrm{Na} \mathrm{CF} / 88^{32}$, em seu artigo 196, a saúde foi inscrita como direito de todos e dever do Estado, que deve ser "garantido mediante politicas sociais e econômicas que visem à redução do risco de doença e de outros agravos e ao acesso universal e igualitário às ações e servi- 
ços para sua promoção, proteção e recuperação". Portanto os direitos à saúde e à justiça social se configuram como direitos sociais garantidos na Carta Magna, um direito público subjetivo e um impostergável dever do Estado ${ }^{33}$.

No entanto, a principal questão não é falta de recursos nem insuficiência no aumento de gastos públicos, pois é difícil encontrar relações consistentes entre aumento nos gastos e resultados que demonstrem a importância de se alocar mais recursos para indivíduos de baixa renda ${ }^{15}$.

Pesquisas apontam que os sistemas de saúde em países de baixa renda com forte orientação para cuidados básicos tendem a ser mais pró-pobres, equitativos e acessíveis. No nível operacional, a maioria dos estudos que comparam serviços que podem ser entregues, como na $\mathrm{AB}$ ou os especializados, mostra que o uso de médicos de cuidados básicos reduz os custos e aumenta a satisfação do usuário, sem efeitos adversos na qualidade dos cuidados ou nos resultados do usuário ${ }^{7}$. As constatações, embora pareçam contraditórias, dependem do contexto e das condições em que são analisadas. Por exemplo, se em países com pouco ou elevado nível de desenvolvimento, se em países com boa ou fraca estrutura de governança pública.

Ao conjugar os resultados da análise de correspondência aos resultados sobre gastos e infraestrutura, na regressão quantílica se verificou que municípios de regiões com menores índices econômicos e sociais recebem menos recursos per capita e possuem, proporcionalmente, mais unidades nas tipologias reprovada (1), rudimentar (2) e restrita (3), o que reflete, ainda mais, as desigualdades socioeconômicas.

Portanto, por meio da análise de correspondência evidenciou-se a relação positiva entre gastos e tipologia da infraestrutura, porém há outra questão relevante: verificar como se dá essa relação nos municípios, considerando-se os objetivos definidos na CF/88.

A análise por meio da regressão quantílica estabeleceu a relação entre variáveis representativas de gastos e a tipologia da infraestrutura básica local de saúde. Verificou-se o sentido de cada relação, a magnitude e a significância, com estimação dos coeficientes para os quantis $0,10,0,50$ e 0,95.

Nessa etapa, obtiveram-se resultados para cada quantil de interesse ao fornecer informações sobre mudanças na distribuição da variável dependente, o que facilitou a interpretação dos resultados para um conjunto de dados que apresentem assimetria, como a variável escore_final, com possibilidade de análise da relação em re- giões centrais da distribuição (por meio da mediana) e nas caudas da distribuição condicional, neste caso as tipologias 1,2 e $5^{22-24}$. Foram analisados os efeitos das variáveis representativas de gasto sobre o escore final.

Para facilitar o entendimento dos efeitos das variáveis de gastos sobre a tipologia, foram sintetizados os resultados referentes a cada variável por quantil, excluindo-se os resultados não significativos ( $\mathrm{p}$-valor acima de 10\%). O Quadro 3 evidencia que os gastos, em geral, influem positivamente na tipologia, com resultado semelhante ao da AC, pois todos os coeficientes com significância (p-valor menor que 10\%) apresentaram sinal positivo. A hipótese diretriz pode ser considerada convergente com os resultados apresentados para as variáveis despesas correntes, despesas administrativas, despesa federal na $A B$, despesa federal total em saúde e despesa total em saúde, considerando-se as variáveis que apresentaram coeficientes com significância ( $p$-valor menor que $10 \%$ ) no quantil 0,5 nos dois ciclos.

Assim, há evidência entre as variáveis de gasto e infraestrutura de maneira positiva, o que converge para o entendimento de que a alocação de mais recursos leva à melhor infraestrutura. No entanto, há grandes disparidades regionais e sociais no Brasil, refletindo-se nas políticas de saúde, especialmente na $A B$, observando-se que, nas regiões Norte e Nordeste, há proporções maiores de estruturas nas tipologias reprovada (1), rudimentar (2) e restrita (3).

Constatou-se também que as variáveis referentes a gastos se relacionam à infraestrutura, mas em países em desenvolvimento, onde as instituições orçamentárias são deficientes, o aumento de gastos, exclusivamente, não garante melhoria nos resultados ${ }^{6,7,15}$. Outros trabalhos demonstram que, em países onde há boa governança e as instituições orçamentárias são bem definidas ou efetivadas, há grandes possibilidades de os gastos se relacionarem diretamente aos resultados $^{8,15,18}$.

Os resultados desta investigação corroboram outros estudos $^{17,18}$, ao constatarem que, mesmo alocando mais recursos que outros países, o Brasil possui indicadores de resultados piores nas políticas de saúde, o que converge para o posicionamento de autores que defendem que a governança orçamentária pode exercer papel fundamental na melhoria da gestão e, consequentemente, na melhor alocação de recursos em saúde, sobretudo na infraestrutura da $\mathrm{AB}$.

Observe-se ainda que essa infraestrutura não deixa de refletir a alocação de recursos: há muni- 


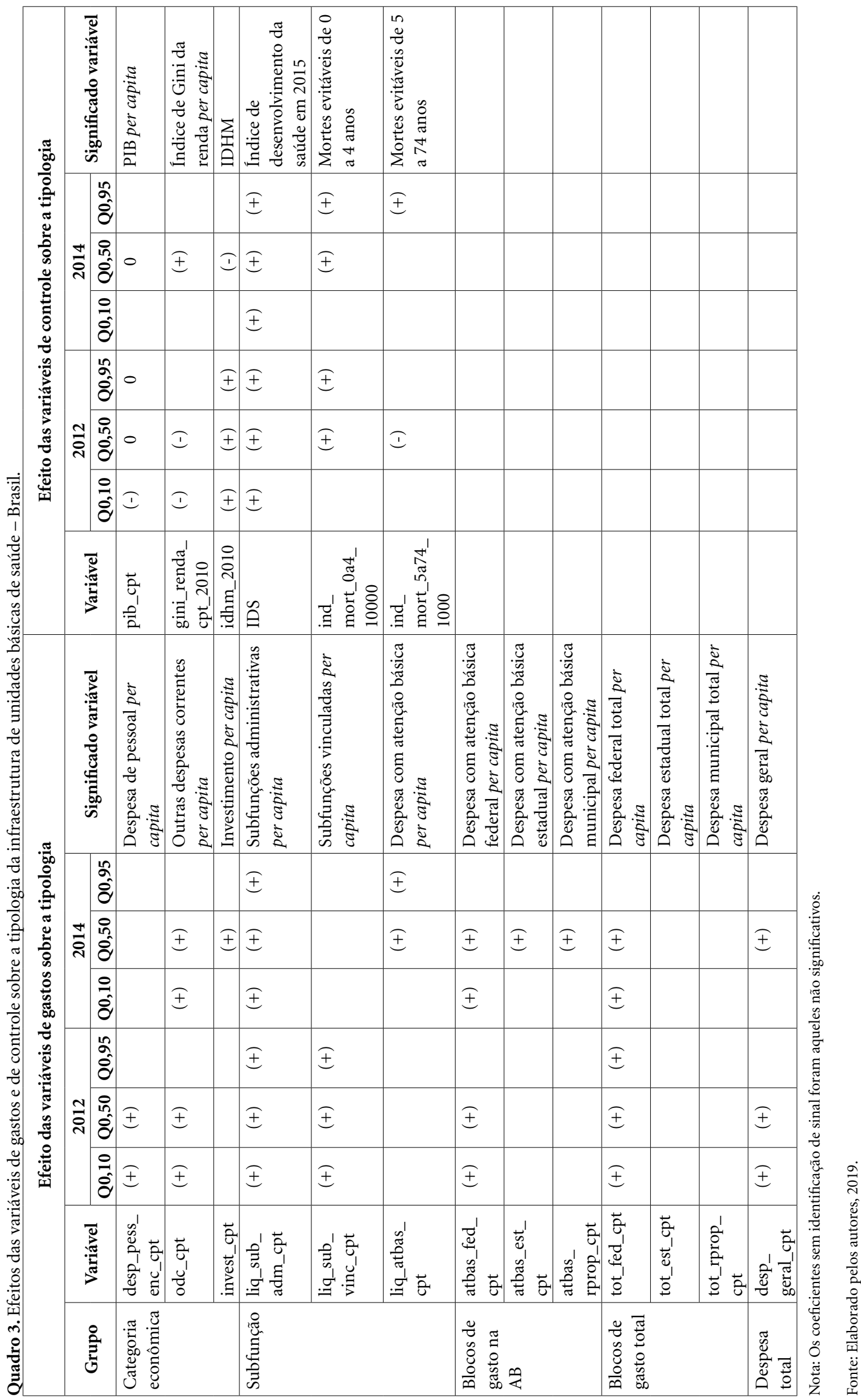


cípios que alocam menos de $\mathrm{R} \$ 50,00$ per capita anuais na $\mathrm{AB}$, ao passo que outros alocam valores superiores a R\$3.500,00. Em 2014, a média alocada por municípios do Pará foi de $\mathrm{R} \$ 217,00$, enquanto a média apresentada por Goiás foi de R\$ 752,00 e a de São Paulo foi de R\$ 722,00.

\section{Análise do comportamento das variáveis de controle}

Em suma, evidenciou-se que no ciclo 1 o tamanho da população está associado negativamente à tipologia, o IDS está associado positivamente à tipologia, o índice de mortes evitáveis de 0 a 4 anos está associado positivamente à tipologia, ao passo que o índice de mortes evitáveis de 5 a 74 anos está associado negativamente (Quadro 3). Ressalta-se que o PIB e o índice de Gini da renda não tiveram resultados claros quando analisados em relação à tipologia.

Ao se verificar que o IDS se relaciona positivamente com a tipologia, nota-se que o desempenho da saúde segue a lógica de alocação de recursos, ou seja, onde se aloca mais recursos há melhor infraestrutura e o desempenho também é melhor.

Evidencia-se ainda que o IDHM se relaciona positivamente à tipologia, refletindo a mesma lógica do IDS, ou seja, alocam-se mais recursos onde o IDHM é melhor, o que leva a uma infraestrutura também melhor.

Embora se esperasse que os índices de mortalidade estivessem relacionados negativamente à tipologia, não é possível estabelecer relação porque esses resultados dependem de outros níveis de atenção.

Saliente-se que o artigo 17 da Lei Complementar no $141 / 2012^{34}$ determina que o rateio dos recursos da União vinculados às ações e serviços públicos de saúde (ASPS) e repassados aos estados, ao Distrito Federal e aos municípios deve observar as necessidades de saúde da população, as dimensões epidemiológica, demográfica, socioeconômica, espacial e de capacidade de oferta de ASPS, de forma a atender os objetivos do inciso II do $\$ 3 .^{\circ}$ do artigo $198 \mathrm{da} \mathrm{CF} / 88^{32}$, com vistas à progressiva redução das disparidades regionais.

Pesquisas mostram que programas de cuidados básicos destinados a melhorar a saúde em populações desfavorecidas em países menos desenvolvidos conseguiram reduzir as lacunas em saúde entre as populações socialmente privadas e as mais favorecidas socialmente ${ }^{35}$. Isso evidencia a necessidade de uma política de saúde com vistas à melhoria das condições de infraestrutura da $\mathrm{AB}$, priorizando gastos do orçamento federal nas regiões com grandes desigualdades socioeconômicas.

\section{Conclusões}

Sob a perspectiva explicativa, este artigo relacionou variáveis por meio da AC e da RQ ao mostrar que "o maior dispêndio de recursos financeiros per capita leva a uma melhor infraestrutura".

Quanto à tipologia da infraestrutura básica local de saúde nos municípios brasileiros, constatou-se que a maioria das UBS está classificada na tipologia regular (4) (51,2\% no ciclo 1 e 76,8\% no ciclo 2), pois essas UBS possuem equipes de saúde da família e saúde bucal que, com melhoria da infraestrutura, alcançariam o padrão de referência (5).

As correspondências entre a tipologia e as variáveis representativas dos gastos - gastos federais na $A B$, gastos federais totais na saúde e gastos totais em saúde - mostram que tipologias inferiores estão relacionadas a menores gastos per capita nessas variáveis e vice-versa.

De forma semelhante, os resultados da RQ tendem a ser convergentes com a hipótese diretriz para as variáveis despesas correntes, despesas administrativas, despesa federal na $A B$, despesa federal total e despesa total em saúde, considerando-se as variáveis que apresentaram coeficientes com significância de pelo menos $10 \%$ no quantil 0,5 nos dois ciclos.

A evidência de que as variáveis de gasto estão relacionadas à infraestrutura de maneira positiva permite entender que alocar mais recursos leva a uma melhor infraestrutura. Todavia, há grandes disparidades regionais e sociais no Brasil, o que se reflete também nas políticas de saúde, especialmente na AB. Nas regiões Norte e Nordeste são apresentadas tipologias reprovada (1), rudimentar (2) e restrita (3).

Mais uma vez, conclui-se aqui a necessidade de melhorar a governança dos recursos da saúde, o que é evidenciado ao se verificar que as regiões com condições socioeconômicas inferiores (PIB per capita e IDHM menores) possuem infraestruturas de $\mathrm{AB}$ também inferiores.

Assim, observou-se que o IDHM está relacionado positivamente à tipologia, refletindo a mesma lógica do desempenho da saúde (IDS), alocando-se mais recursos onde o IDHM é melhor. Nesse contexto, é importante salientar as pesquisas que mostram programas de cuidados básicos destinados a melhorar a saúde em populações desfavorecidas em países menos desen- 


\section{Colaboradores}

HK Kashiwakura: responsável por todos os testes quantitativos, organizou toda a base de dados da pesquisa, bem como participou na redação do texto. AO Gonçalves: liderou o processo de pesquisa e organizou todo o texto para a elaboração deste artigo. RR Azevedo: conferiu todos os testes quantitativos e realizou os ajustes necessários, bem como revisou todo o texto do artigo. A Nunes: definiu em conjunto com os autores as discussões dos resultados e conclusões do artigo. CAT Silva: adequou corretamente o conteúdo metodológico no artigo. nações que alocam menos recursos nas políticas de saúde, o que converge para o posicionamento dos defensores da governança orçamentária, haja vista que essa pode exercer papel fundamental na melhoria da gestão e, consequentemente, na melhor alocação de recursos em saúde.

\section{Referências}

1. Guerra M. Modelo de alocação de recursos do sistema único de saúde para organizações hospitalares: serviços de alta complexidade [tese]. Brasília: Universidade de Brasília; 2013.

2. Cordeiro H. Descentralização, universalidade e equidade nas reformas da saúde. Cien Saude Colet 2001; 6(2):319-328.

3. Brasil. Ministério da Saúde (MS). Secretaria de Atenção à Saúde. Departamento de Atenção Básica. $M a-$ nual de estrutura física das unidades básicas de saúde: saúde da família. 2. ed. Brasília: MS; 2008.

4. Organização Panamericana de Saúde. Health in the Americas, 2007. Washington (DC): OPAS; 2007.

5. Piola SF, Servo LM, de Sá EB, de Paiva AB. Financiamento do Sistema Único de Saúde - Trajetória recente e cenários para o futuro. Anal. Econ. 2012; 30.

6. Filmer D, Hammer J, Pritchett L. Health policy in poor countries: weak links in the chain [Research working paper no WPS 1874]. Washington (DC): World Bank; 1997. Disponível em: http://documents.worldbank. org.

7. Atun R. What are the advantages and disadvantages of restructuring a health care system to be more focused on primary care services. Copenhagen: World Health Organization Publisher; 2004. Disponível em: http:// www.euro.who.int/document/e82997.pdf.

8. Rajkumar AS, Swaroop V. Public spending and outcomes: does governance matter? J Dev Econ 2008; 86(1):96-111. 
9. David GC. Atenção primária nos municípios brasileiros entre 2007-2010: desempenho, gasto, eficiência e disparidades [dissertação]. Brasília: Universidade de Brasília; 2015.

10. Varela PS, Farina MC. Relação entre gastos com saúde, índice de esforços da atenção básica em saúde e tipologia da estrutura do sistema de saúde dos municípios do estado de São Paulo. Rev Adm Mackenzie 2007; 8(3):153-172.

11. Giovanella L, Bousquat A, Fausto MC, Fusaro E, Mendonca M, Gagno J. Tipologia das unidades básicas de saúde brasileiras. Novos Caminhos 2015 [acessado em 2017 Abr 12]; (5). Disponível em: http://www.resbr. net.br/wp-content/uploads/2015/09/NovosCaminhos05_ValeEste.pdf.

12. Scatena JH, Tanaka OY. Distribuição dos estabelecimentos de saúde no Brasil: para qual modelo caminhamos? Rev Adm Pública 1998; 32(4):7-25.

13. Guimarães C, Amaral P, Simões R. Rede urbana da oferta de serviços de saúde: uma análise multivariada macrorregional - Brasil; 2002. In: Anais do XV Encontro Nacional de Estudos Populacionais, ABEP; 2006 Set 18-22; Caxambu. p. 1-8.

14. Simões R, Guimarães C, Godoy N, Velloso T, Araújo T, Galinari R, Chein F. Rede urbana da oferta de serviços de saúde: uma análise espacial multivariada para Minas Gerais. In: Seminário sobre a Economia Mineira; 2004. Belo Horizonte: CEDEPLAR, UFMG. p. 1-27.

15. World Bank. Making services work for poor people: world development report 2003. Washington (DC): World Bank; 2003.

16. Savigny D, Adam T, editors. Systems thinking for health systems strengthening. Geneva: World Health Organization; 2009.

17. Ferraz OL, Vieira FS. Direito à saúde, recursos escassos e equidade: os riscos da interpretação judicial dominante. Rev Bras Ci Soc 2009; 52(1):223-51.

18. Banco Mundial. Governança no sistema único de saúde (SUS) do Brasil: melhorando a qualidade do gasto público e gestão de recursos. Brasília: Banco Mundial; 2007. p. 42.

19. Greenacre MJ. Practical correspondence analysis. In: Barnett V, organizador. Looking at Multivariate Data. New York: J. Wiley \& Sons; 1981. p. 81-107.

20. Hoffman DL, Franke GR. Correspondence analysis: graphical representation of categorical data in marketing research. J Mark Res 1986; 23(3):213-27.

21. Gouvêa V. Análise de correspondências: textos didáticos. Rio de Janeiro: ENCE/IBGE; 1990.

22. Koenker R. Quantile regression. New York: Cambridge University Press; 2005.

23. Hao L, Naiman DQ. Quantile regression. California: Sage Publications; 2007.

24. Santos PM. Regressão quantílica aplicada ao estudo de seleção genômica para características assimétricas de suinos [dissertação]. Viçosa: Universidade Federal de Viçosa; 2016.

25. World Bank. World Development Report 2004: making services work for poor people. London, Oxford University Press [acessado 2019 jul 12]; 10(1). World Bank; 2004. Disponível em: www.worldbank.org.
26. Maynard A, Bloor K. Our certain fate: rationing in health care. London: Office of Health Economics; 1998.

27. Ostrom E. Governing the commons: the evolution of institutions for collective action. New York: Cambridge university press; 1990.

28. Agrawal A. Sustainable governance of common-pool resources: context, methods, and politics. Annu Rev Anthropol 2003; 32(1):243-62.

29. Barcelos CLK. Governança orçamentária e mudança institucional: o caso da norma geral de direito financeiro - Lei n. ${ }^{\circ}$ 4.320/64 [tese]. Brasília: Universidade de Brasília; 2012.

30. Kringos DS, Boerma WG, Bourgueil Y, Cartier T, Hasvold T, Hutchinson A, Lember M, Oleszczyk M, Pavlic DR, Svab I, Tedeschi P. The European primary care monitor: structure, process and outcome indicators. BMC Fam Pract 2010; 11(1):81.

31. Declaração de Alma-Ata. Conferência Internacional sobre cuidados primários de saúde; 6-12 de setembro de 1978; Alma-Ata; USSR. In: Ministério da Saúde (BR). Secretaria de Políticas de Saúde. Projeto Promoção da Saúde. Declaração de Alma-Ata; Carta de Ottawa; Declaração de Adelaide; Declaração de Sundsvall; Declaração de Santafé de Bogotá; Declaração de Jacarta; Rede de Megapaíses; Declaração do México. Brasília (DF): Ministério da Saúde; 2001. p. 15.

32. Brasil. Constituição da República Federativa do Brasil. Brasília: Senado Federal; 1988.

33. Noronha JC, Pereira TR. Princípios do Sistema de Saúde Brasileiro. In: Fundação Oswaldo Cruz. A saúde no Brasil em 2030 - prospecção estratégica do sistema de saúde brasileiro: organização e gestão do sistema de saúde [página na Internet]. Rio de Janeiro: Fiocruz/Ipea/MS/ SAE-PR; 2013. [acessado 2019 jul 12]. Disponível em: https://static.scielo.org/scielobooks/98kjw/pdf/noronha-9788581100173.pdf

34. Brasil. Lei Complementar $n^{\circ} 141$, de 13 de janeiro de 2012. Brasília: Diário Oficial da União 2012; 16 Jan.

35. Starfield B. Atenção primária: equilíbrio entre necessidades de saúde, serviços e tecnologia. Brasília: Unesco; 2005.

Artigo apresentado em 29/05/2019

Aprovado em 20/04/2020

Versão final apresentada em 22/04/2020

Editores-chefes: Romeu Gomes, Antônio Augusto Moura da Silva 


\section{ERRATA}

Ciência \& Saúde Coletiva

volume 26 suplemento $2-2021$

No artigo Retrato da atenção básica no Brasil: gastos e infraestrutura em municípios brasileiros, DOI: $10.1590 / 1413-81232021269.2 .37112019$

p. 3403,

onde se lê:

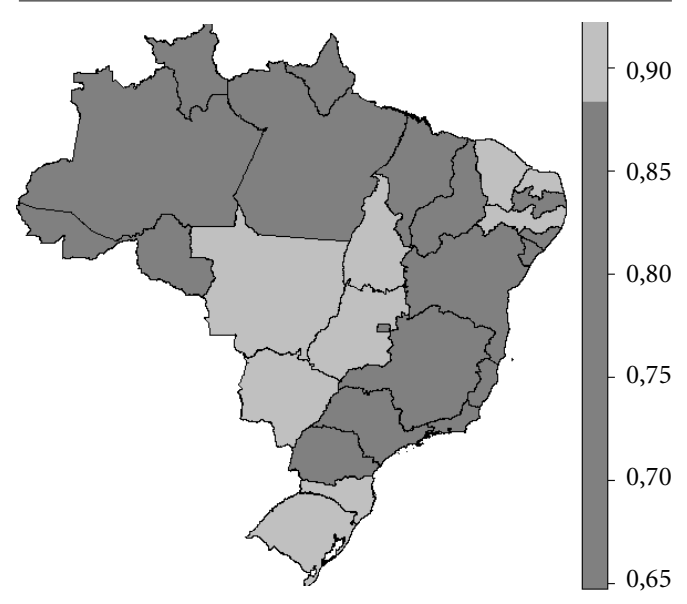

Figura 1. Distribuição da tipologia por média de escore das UBS por estado (ciclo 2).

Legenda: A escala de cores à direita representa a média dos escores das UBS dos municípios por estado.

Fonte: Elaborado pelos autores, 2019.

\section{leia-se:}

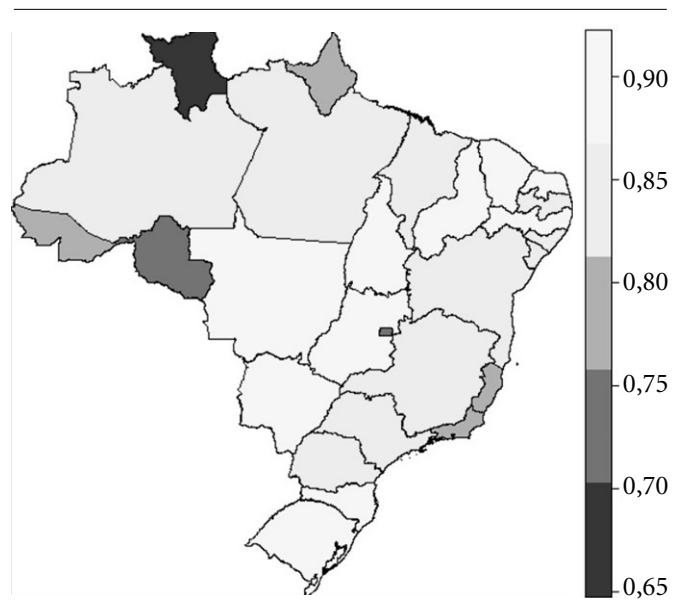

Figura 1. Distribuição da tipologia por média de escore das UBS por estado (ciclo 2).

Legenda: A escala de cores à direita representa a média dos escores das UBS dos municípios por estado.

Fonte: Elaborado pelos autores, 2019. 